\title{
PRODUCT INTEGRATION FOR VOLTERRA INTEGRAL EQUATIONS OF THE SECOND KIND WITH WEAKLY SINGULAR KERNELS
}

\author{
ANNAMARIA PALAMARA ORSI
}

\begin{abstract}
We introduce a new numerical approach for solving Volterra integral equations of the second kind when the kernel contains a mild singularity. We give a convergence result. We also present numerical examples which show the performance and efficiency of our method.
\end{abstract}

\section{INTRODUCTION}

We consider Volterra integral equations of the second kind,

$$
y(x)=g(x)+\int_{a}^{x} p(x, s) K(x, s, y(s)) d s, \quad a \leq x<\infty,
$$

where the kernel $p$ is weakly singular and the given functions $g$ and $K$ are assumed to be sufficiently smooth in order to guarantee the existence and uniqueness of a solution $y \in C[a, b]$ (see, for instance, [3], [4, (§6.9)], [10, (Thm. 1.3.2)], [11]).

Typical forms of $p(x, s)$ are

$$
\begin{gathered}
p(x, s)=|x-s|^{-\alpha}, \quad 0<\alpha<1, \\
p(x, s)=\log |x-s| .
\end{gathered}
$$

For Volterra equations with bounded kernels, the smoothness of the kernel and of the forcing function $g(x)$ determines the smoothness of the solution on the closed interval $[a, X]$, with $X>a$. If we allow weakly singular kernels, then the resulting solutions are typically nonsmooth at the initial point of the interval of integration, where their derivatives become unbounded. Some results concerning the behavior of the exact solutions of equations of type (1) are given in [10, (1.3.5)], [22, 23].

Numerical methods for solving Abel equations of the second kind, i.e., of type (1) with kernel (i), have been considered by several authors (see [10, Ch. 6]). Such methods are of two kinds:

1. discretization methods derived under the assumption that the solution $y$ is smooth on the closed interval $[a, X]$ (see for instance $[12,31]$ );

2. methods for equations (1) with nonsmooth solutions, taking into account their singular behavior in the neighborhood of the point $s=a$ (see for instance $[1,6,9,14,24])$.

Received by the editor December 30, 1991 and, in revised form, September 21, 1993 and November 29, 1994.

1991 Mathematics Subject Classification. Primary 65R20, 65D32.

This work was sponsored by the "Ministero dell'Università e della Ricerca Scientifica e Tecnologica" of Italy.

(C)1996 American Mathematical Society 
In the numerical examples presented in these papers the case of practical importance, $\alpha=1 / 2$, is usually considered. A survey of the literature on Abel-type integral equations may be found in [16].

In this paper we introduce a new method for solving equation (1). This method allows us to overcome the difficulty caused by the poor behavior of the solution $y(s)$ at the initial point $s=a$.

Given a relatively short interval $[a, b]$, we first solve the problem

$$
y(x)=g(x)+\int_{a}^{x} p(x, s) K(x, s, y(s)) d s, \quad a \leq x \leq b,
$$

by a Nyström-type method based upon a whole-interval product integration rule of interpolatory type, which integrates exactly the kernel $p$.

After the initial interval, the bad behavior of the derivative of $y$ is of less significance. We then solve the problem

$$
y(x)=g_{1}(x)+\int_{b}^{x} p(x, s) K(x, s, y(s)) d s, \quad b \leq x<\infty,
$$

with

$$
g_{1}(x)=g(x)+\int_{a}^{b} p(x, s) K(x, s, y(s)) d s,
$$

by a standard step-by-step method for regular solutions. Since the computation of $g_{1}(x)$ depends on the starting approximation of $y(x), x \in[a, b]$, the two methods have to be regarded as paired.

The ideas underlying the starting procedure for the numerical solution of problem (2) are well known and have already been applied to weakly singular Fredholm integral equations of the second kind, although they have not been used in this context before. In $\S 2$ we describe our starting method, and in $\S 3$ give uniform convergence results in the linear case. In $\S 4$ we apply our starting method, together with a classical scheme for the numerical solution of (3), to some test equations and compare our numerical results with the ones available in the literature and obtained by alternative methods.

\section{The PRODUCT INTEGRATION RULE}

In this section we describe the Nyström-type method used to solve equation (2) numerically. For convenience, we assume $[a, b]=[-1,1]$, without any loss of generality:

$$
y(x)=g(x)+\int_{-1}^{x} p(x, s) K(x, s, y(s)) d s, \quad-1 \leq x \leq 1 .
$$

Having chosen $N+1$ distinct points $\left\{x_{n}\right\}_{n=0}^{N}$ in the interval [-1,1], we collocate the equation (5) at the nodes $\left\{x_{n}\right\}_{n=0}^{N}$ :

$$
y\left(x_{n}\right)=g\left(x_{n}\right)+\int_{-1}^{x_{n}} p\left(x_{n}, s\right) K\left(x_{n}, s, y(s)\right) d s, \quad n=0,1, \ldots, N .
$$

Then we use the Lagrange interpolation polynomial

$$
L_{N}(K ; s)=\sum_{j=0}^{N} l_{N, j}(s) K\left(x_{n}, x_{j}, y\left(x_{j}\right)\right)
$$


to approximate $K\left(x_{n}, s, y(s)\right)$ and obtain the following method:

$$
y_{N, n}=g\left(x_{n}\right)+\sum_{j=0}^{N} w_{n, j} K\left(x_{n}, x_{j}, y_{N, j}\right), \quad n=0,1, \ldots, N
$$

with $w_{n, j}=\int_{-1}^{x_{n}} p\left(x_{n}, s\right) l_{N, j}(s) d s$.

To construct the coefficients $w_{n, j}$, we use an algorithm introduced in [26]. This algorithm requires existence and knowledge of the modified moments

$$
\mu_{k}\left(x_{n}\right)=\int_{-1}^{x_{n}} p\left(x_{n}, s\right) P_{k}(s) d s, \quad k=0,1, \ldots,
$$

corresponding to the weakly singular kernel $p(x, s)$, where $\left\{P_{k}\right\}$ is a system of polynomials satisfying a three-term recurrence relation. Recurrence formulas for the modified moments can be found in [28] and [4] for various kernels. In [28] the polynomials $\left\{P_{k}\right\}$ used are the Chebyshev polynomials of the first kind, in [4, pp. 560-561], for kernels of type (i) and (ii), the Legendre polynomials.

Once the weights $\left\{w_{n, j}\right\}$ have been obtained, we compute the approximate solution values $y_{N, n}, n=0,1, \ldots, N$, as solution of the system (8). In the linear case the system (8) is solved by Gaussian elimination; in the nonlinear case we compute $\left\{y_{N, n}\right\}_{n=0}^{N}$ from (8) by using the nonlinear systems solver CO5NBF of the NAG library.

\section{Starting Method: CONVERGENCE}

Throughout this section, the symbol $C$ stands for a positive constant taking on different values on different occurrences.

In our convergence analysis we examine the linear test equation

$$
y(x)=g(x)+\int_{-1}^{x} p(x, s) y(s) d s, \quad-1 \leq x \leq 1,
$$

and assume that the forcing function $g \in C[-1,1]$, and that the kernel $p$ is weakly singular of the form (i) or (ii). Then the equation (10) has a unique solution $y \in C[-1,1]$ that may be expected to have unbounded derivatives at the endpoint $x=-1$.

If, for a given mesh $\left\{x_{j}\right\}_{j=0}^{N}$, we apply the method (8) to the test equation (10), we obtain as approximate solution $y_{N}(x)$ the following Nyström interpolant:

$$
y_{N}(x)=g(x)+\sum_{j=0}^{N} w_{j}(p ; x) y_{N}\left(x_{j}\right), \quad \text { where } w_{j}(p ; x)=\int_{-1}^{x} p(x, s) l_{N, j}(s) d s .
$$

The method is said to be convergent of order $r$ in $[-1,1]$ if and only if for $N$ sufficiently large there exists a constant $C>0$ independent of $N$ such that

$$
\left\|y(x)-y_{N}(x)\right\|_{\infty} \leq C N^{-r} .
$$

In order to examine the uniform convergence of the approximate solution $y_{N}(x)$ to the exact solution $y(x)$ of $(10)$, notice that

$$
y(x)-y_{N}(x)=\sum_{j=0}^{N} w_{j}(p ; x)\left\{y\left(x_{j}\right)-y_{N}\left(x_{j}\right)\right\}+t_{N}(p, y ; x),
$$


where $t_{N}(p, y ; x)$ is the local truncation error defined by

$$
t_{N}(p, y ; x)=\int_{-1}^{x} p(x, s) y(s) d s-\sum_{j=0}^{N} w_{j}(p ; x) y\left(x_{j}\right) .
$$

Hence, we obtain

$$
\left\|y-y_{N}\right\|_{\infty} \leq\left\|\left(I-A_{N}\right)^{-1}\right\|_{\infty}\left\|t_{N}\right\|_{\infty},
$$

where $A_{N}$ is the linear operator defined by

$$
\left\{\begin{array}{l}
A_{N}: C[-1,1] \rightarrow C[-1,1], \\
A_{N} f(x)=\sum_{j=0}^{N} w_{j}(p ; x) f\left(x_{j}\right), \quad f \in C[-1,1], x \in[-1,1] .
\end{array}\right.
$$

First we investigate the convergence properties of the underlying product quadrature rule.

Theorem 1. Let $\left\{x_{j}\right\}_{j=0}^{N}$ be the zeros of the $(N+1)$ st-degree member of a set of polynomials that are orthogonal on $[-1,1]$ with respect to the weight function

$$
\omega(s)=u(s)(1-s)^{\bar{\alpha}}(1+s)^{\bar{\beta}}, \quad-1<\bar{\alpha} \leq \frac{3}{2}, \bar{\beta} \geq-\frac{1}{2} .
$$

Here, $u(x)$ is positive and continuous in $[-1,1]$ and the modulus of continuity $\varphi$ of $u$ satisfies $\int_{0}^{1} \varphi(u, \delta) \frac{d \delta}{\delta}<\infty$. Let $L_{N}(f ; s)$ denote the interpolating polynomial of degree $\leq N$ that coincides with the function $f$ at the nodes $\left\{x_{j}\right\}_{j=0}^{N}$. Moreover, suppose $p(x, s)$ is a kernel of type (i) or (ii). Then, for every function $f$ containing only endpoint singularity of the type $(1+s)^{\sigma}, \sigma>-1$ (not an integer), and in particular for every function $f \in C[-1,1]$, there holds

$$
\lim _{N \rightarrow \infty}\left\|\int_{-1}^{x} p(x, s) f(s) d s-\int_{-1}^{x} p(x, s) L_{N}(f ; s) d s\right\|_{\infty}=0 .
$$

In particular, we have the bounds

$$
\begin{gathered}
\left\|t_{N}\left(|x-s|^{-\alpha}, f ; x\right)\right\|_{\infty}=O\left\{(N+1)^{-2-2 \sigma+2 \alpha} \log (N+1)\right\}, \quad 0<\alpha<1 \\
\left\|t_{N}(\log |x-s|, f ; x)\right\|_{\infty}=O\left\{(N+1)^{-2-2 \sigma} \log ^{2}(N+1)\right\}
\end{gathered}
$$

Proof. Since

$$
\begin{aligned}
\left|t_{N}(p(x, s), f ; x)\right| & \leq \int_{-1}^{x}|p(x, s)|\left|f(s)-L_{N}(f ; s)\right| d s \\
& \leq \int_{-1}^{1}|p(x, s)|\left|f(s)-L_{N}(f ; s)\right| d s
\end{aligned}
$$

the bounds (15) and (16) are an immediate consequence of Theorems 5 and 7 in [13].

Now we investigate the behavior of the first term $\left\|\left(I-A_{N}\right)^{-1}\right\|_{\infty}$ in the righthand side of (11). To this end, some preliminary lemmas are needed.

Lemma 1. For a given set of nodes $\left\{x_{j}\right\}_{j=0}^{N}$ defined as in Theorem 1 with the restriction $-\frac{1}{2}<\bar{\alpha}, \bar{\beta}<\frac{3}{2}$, let $l_{N, j}(s)$ denote the corresponding $j$ th fundamental 
Lagrange polynomial. Fix a subinterval $[c, d] \subseteq[-1,1]$. Then there exists a positive number $C$ and $a q>1$ such that

$$
\sup _{N} \sum_{j=0}^{N}\left|\int_{c}^{d} p(d, s) l_{N, j}(s) d s\right| \leq C\left[\int_{c}^{d}|p(d, s)|^{q} d s\right]^{1 / q}
$$

for all $p \in L_{q}$ with $\|p\|_{q}=\left[\int_{-1}^{1}|p(d, s)|^{q} d s\right]^{1 / q}$.

Proof. First of all, notice that

$$
\sum_{j=0}^{N}\left|\int_{c}^{d} p(d, s) l_{N, j}(s) d s\right|=\sup _{f \in B}\left|\sum_{j=0}^{N} \int_{c}^{d} p(d, s) l_{N, j}(s) d s f\left(x_{j}\right)\right|,
$$

where $B=\left\{f: f \in C[-1,1],\|f\|_{\infty}=1\right\}$. Then we have

$$
\begin{aligned}
& \sum_{j=0}^{N}\left|\int_{c}^{d} p(d, s) l_{N, j}(s) d s\right|=\sup _{f \in B}\left|\int_{c}^{d} \sum_{j=0}^{N} l_{N, j}(s) f\left(x_{j}\right) p(d, s) d s\right| \\
& \quad \leq \sup _{f \in B} \int_{c}^{d}\left|\left\{\sum_{j=0}^{N} l_{N, j}(s) f\left(x_{j}\right)\right\} p(d, s)\right| d s \\
& \quad \leq \sup _{f \in B}\left[\int_{c}^{d}\left|\sum_{j=0}^{N} l_{N, j}(s) f\left(x_{j}\right)\right|^{q^{\prime}} d s\right]^{1 / q^{\prime}}\left[\int_{c}^{d}|p(d, s)|^{q} d s\right]^{1 / q} \\
& \quad \leq \sup _{f \in B}\left[\int_{-1}^{1}\left|\sum_{j=0}^{N} l_{N, j}(s) f\left(x_{j}\right)\right|^{q^{\prime}} d s\right]^{1 / q^{\prime}}\left[\int_{c}^{d}|p(d, s)|^{q} d s\right]^{1 / q}
\end{aligned}
$$

for all $p \in L_{q}$ with $q, q^{\prime}>1$ such that

$$
\frac{1}{q}+\frac{1}{q^{\prime}}=1
$$

From Theorem 1 in [27], under the assumptions of this lemma, we have

$$
\sup _{N}\left\|\sum_{j=0}^{N} l_{N, j}(s) f\left(x_{j}\right)\right\|_{q^{\prime}} \leq C\|f\|_{\infty}
$$

for every bounded function $f$, and $0<q^{\prime}<\infty$. Therefore, the bound (18) follows.

Lemma 2. Let the same notations and the same set of nodes be assumed as in Lemma 1. Moreover, let the kernel p satisfy

$$
\left\{\begin{array}{l}
p \in L_{q}, \quad q>1 \\
\lim _{x^{\prime} \rightarrow x}\left\|p\left(x^{\prime}, s\right)-p(x, s)\right\|_{q}=0
\end{array}\right.
$$

for all $x \in[-1,1]$. Then

$$
\lim _{x^{\prime} \rightarrow x} \sup _{N} \sum_{j=0}^{N}\left|w_{j}\left(p^{\prime} ; x^{\prime}\right)-w_{j}(p ; x)\right|=0
$$

for all $x \in[-1,1]$, where $p^{\prime}=p\left(x^{\prime}, s\right)$. 
Proof. In order to prove (20), notice that, for all $p, p^{\prime} \in L_{q}$,

$$
\begin{aligned}
\sup _{N} & \sum_{j=0}^{N}\left|w_{j}\left(p^{\prime} ; x^{\prime}\right)-w_{j}(p ; x)\right| \\
& =\sup _{N} \sum_{j=0}^{N}\left|\int_{-1}^{x} l_{N, j}(s)\left\{p\left(x^{\prime}, s\right)-p(x, s)\right\} d s+\int_{x}^{x^{\prime}} p\left(x^{\prime}, s\right) l_{N, j}(s) d s\right| \\
& \leq \sup _{N}\left\{\sum_{j=0}^{N}\left|\int_{-1}^{x} l_{N, j}(s)\left\{p\left(x^{\prime}, s\right)-p(x, s)\right\} d s\right|+\sum_{j=0}^{N}\left|\int_{x}^{x^{\prime}} p\left(x^{\prime}, s\right) l_{N, j}(s) d s\right|\right\} \\
& \leq C\left\{\left[\int_{-1}^{x}\left|p\left(x^{\prime}, s\right)-p(x, s)\right|^{q} d s\right]^{1 / q}+\left[\int_{x}^{x^{\prime}}\left|p\left(x^{\prime}, s\right)\right|^{q} d s\right]^{1 / q}\right\} \\
& \leq C\left\{\left\|p\left(x^{\prime}, s\right)-p(x, s)\right\|_{q}+\left[\int_{x}^{x^{\prime}}\left|p\left(x^{\prime}, s\right)\right|^{q} d s\right]^{1 / q}\right\},
\end{aligned}
$$

where in the penultimate step we have used Lemma 1. The assertion (20) now follows from (19).

Now we can obtain the following result.

Theorem 2. Let the operator $A_{N}$ be defined as in (12) and the nodes $\left\{x_{j}\right\}_{j=0}^{N}$ chosen as in Lemma 1. If (14), (18) and (20) hold, then for all $N$ sufficiently large there exists a constant $C>0$ independent of $N$ such that

$$
\left\|\left(I-A_{N}\right)^{-1}\right\|_{\infty} \leq C .
$$

Proof. If (14), (18), and (20) hold, by the arguments used in the proof of Lemma 1 in [30] on a collectively compact set of operators, we can deduce that the set $S=\left\{A_{N} f: f \in C[-1,1],\|f\|_{\infty}=1, N \geq 0\right\}$ is a bounded, equicontinuous subset of $C[-1,1]$; then the sequence of bounded operators $\left\{A_{N}\right\}$ is collectively compact.

Moreover, we can show that the operator $A$ defined by

$$
\left\{\begin{array}{l}
A: C[-1,1] \rightarrow C[-1,1], \\
A f(x)=\int_{-1}^{x} p(x, s) f(s) d s
\end{array}\right.
$$

is compact. In fact, Kress in [20] proved that the operator $A^{*}$ defined by

$$
\left\{\begin{array}{l}
A^{*}: C[-1,1] \rightarrow C[-1,1], \\
A^{*} f(x)=\int_{-1}^{1} p(x, s) f(s) d s
\end{array}\right.
$$

is a compact operator on $C[-1,1]$, if the kernel $p$ is defined and continuous for all $x, s \in[-1,1], x \neq s$, and $|p(x, s)| \leq C|x-s|^{-\alpha}, 0 \leq \alpha<1$, for all $x, s \in[-1,1], x \neq$ $s$. In the case $p(x, s)=|x-s|^{-\alpha}, 0<\alpha<1$, it follows that our operator $A$ is 
compact, since we can always write

$$
\begin{aligned}
A f(x)=\int_{-1}^{x} p(x, s) f(s) d s & =\int_{-1}^{1} p^{*}(x, s) f(s) d s \\
\text { with the new kernel } p^{*}(x, s) & = \begin{cases}p(x, s), & s<x, \\
0, & s \geq x,\end{cases}
\end{aligned}
$$

which satisfies the conditions above.

Furthermore, in the case $p(x, s)=\log |x-s|$, we can write

$$
\log |x-s|=\frac{\log |x-s||x-s|^{\alpha}}{|x-s|^{\alpha}}=h(x, s)|x-s|^{-\alpha}, \quad 0<\alpha<1,
$$

where $h(x, s) \in C[-1,1]$ for all $x \in[-1,1]$.

Thus, we conclude that the operator $A$ with kernel (i) or (ii) is compact.

Hence, by Theorem 1.6 in [2] the statement of Theorem 2 follows.

Lemma 3. The kernels $p$ of the form (i) or (ii) fulfill the conditions (19).

Proof. Given $p(x, s)=\frac{1}{(x-s)^{\alpha}}, 0 \leq \alpha<1$, take $q>1$ such that $q \alpha<1$. Then

$$
\left|p\left(x^{\prime}, s\right)-p(x, s)\right|^{q}=\left|\frac{|x-s|^{\alpha}-\left|x^{\prime}-s\right|^{\alpha}}{\left|x^{\prime}-s\right|^{\alpha}|x-s|^{\alpha}}\right|^{q} \leq \frac{C\left|x-x^{\prime}\right|^{\alpha q}}{\left|x^{\prime}-s\right|^{\alpha q}|x-s|^{\alpha q}}
$$

and (see [25, p. 211])

$$
\int_{-1}^{1} \frac{d s}{\left|x^{\prime}-s\right|^{\alpha q}|x-s|^{\alpha q}} \leq \begin{cases}C & \text { if } 2 \alpha q<1 \\ C \log \left|x-x^{\prime}\right| & \text { if } 2 \alpha q=1 \\ \frac{1}{\left|x^{\prime}-x\right|^{2 \alpha q-1}} & \text { if } 2 \alpha q>1\end{cases}
$$

Hence, [19] follows.

From (22) we immediately deduce that also $p(x, s)=\log |x-s|$ fulfills (19).

Finally, we can state the following result.

Theorem 3. Let $y$ be the exact solution of the equation (10). Let $y_{N}$ be the approximate solution obtained by discretizing the integral term of (10) by a product quadrature rule of interpolatory type constructed on a set of distinct nodes $\left\{x_{j}\right\}_{j=0}^{N}$. If the nodes $\left\{x_{j}\right\}_{j=0}^{N}$ are the zeros of the $(N+1)$ st-degree member of a set of polynomials that are orthogonal on $[-1,1]$ with respect to the weight function (13) with $-\frac{1}{2}<\bar{\alpha}, \bar{\beta}<\frac{3}{2}$, and if $p$ is of the form (i) or (ii), then $y_{N}$ converges uniformly to $y$. Moreover, the rate of convergence of $y_{N}$ to $y$ coincides with the one of the basic quadrature rule we choose to approximate the integral term of (10).

The proof follows immediately from the estimate (11) together with Theorems 1 and 2. The bounds (15) and (16) supply an estimate of the rate of convergence.

Remark. Theorem 3 can be easily extended to the case of quadrature rules which include among their nodes the endpoints \pm 1 . In particular, we have the same result if the nodes $\left\{x_{j}\right\}_{j=0}^{N-1}$ are the zeros of the Jacobi polynomial $P_{N}^{(\bar{\alpha}, \bar{\beta})}(x)$, with $-\frac{1}{2}<\bar{\alpha}, \bar{\beta}<7 / 2$, and $x_{N}=1$ (see $\left.[13,27]\right)$. 


\section{NumERICAL EXAMPLES AND DISCUSSION}

The method (8) based on Radau nodes (i.e., on the nodes coinciding with the zeros of the Jacobi polynomial $P_{N}^{(1,0)}(x)$ in addition to the endpoint $\left.x=1\right)$ together with a product Simpson's method on uniform meshes [12] has been implemented to solve the following equations, taken from collections of problems proposed in [10] and $[29]$ :

$$
\begin{aligned}
& y(x)=1-e^{-x}-\frac{1}{\sqrt{\pi}} \int_{0}^{x}(x-s)^{-\frac{1}{2}} y(s) d s \\
& y(x)=\frac{1}{2}\left\{e^{x} \operatorname{erfc}(\sqrt{x})-e^{-x}+2 \sqrt{\pi} F(\sqrt{x})\right\} \\
& F(t)=e^{-t^{2}} \int_{0}^{t} e^{u^{2}} d u \\
& y(x)=\frac{1}{\sqrt{1+x}}+\frac{\pi}{8}-\frac{1}{4} \sin ^{-1} \frac{1-x}{1+x}-\frac{1}{4} \int_{0}^{x}(x-s)^{-\frac{1}{2}} y(s) d s, \\
& y(x)=\frac{1}{\sqrt{1+x}} ; \\
& y(x)=-\frac{1}{\sqrt{\pi}} \int_{0}^{x}(x-s)^{-\frac{1}{2}}\{y(s)-\sin s\}^{3} d s \\
& y(x)=O\left(x^{3} \sqrt{x}\right) \quad \text { as } x \rightarrow 0 .
\end{aligned}
$$

Given an initial steplength $h$, we fix the point $b$ in the open interval $(a, X)$, with $X>a$. Then we first solve the problem (2) by the method (8) based on $N+1$ Radau nodes on the interval $[a, b]$. If the kernel $p$ is of type (i) and the solution $y(s)$ contains only an endpoint singularity of the form $(s-a)^{\sigma}, \sigma>-1$, the order of convergence of this method is $(N+1)^{-2-2 \sigma+2 \alpha} \log (N+1)$ (see $\left.\S 3\right)$. With $b$ fixed, on the interval $[b, X]$ we define the grid $x_{0} \equiv b, x_{n}=x_{0}+n h, n=1,2, \ldots, M, x_{M} \equiv X$, and apply the product Simpson's method [12]. We obtain the approximate values $y_{n}, n=2, \ldots, M$, of

$$
\left\{\begin{array}{l}
y\left(x_{n}\right)=g_{1}\left(x_{n}\right)+\int_{x_{0}}^{x_{n}}\left(x_{n}-s\right)^{-\alpha} K\left(x_{n}, s, y(s)\right) d s, \\
g_{1}\left(x_{n}\right)=g\left(x_{n}\right)+\int_{a}^{x_{0}}\left(x_{n}-s\right)^{-\alpha} K\left(x_{n}, s, y(s)\right) d s, \quad \alpha \leq s \leq x_{0} .
\end{array}\right.
$$

Since the solution $y(x)$ is smooth in the closed interval $[b, X]$ (see [23]), this method converges like $h^{4-\alpha}$ (see [12]).

The starting values $y_{0}$ and $y_{1}$ are evaluated by using the Nyström interpolant on the interval $\left[a, x_{0}\right]$ and the Simpson's formula constructed on the three points $x_{0}-h, x_{0}, x_{0}+h$.

An important problem is the choice of $b$ : the interval $[a, b]$ must be small in order to reduce the computational cost of the pair of methods; in fact the method (8) requires the solution of a full $(N+1) \times(N+1)$ system of equations, whereas the step-by-step product Simpson's method solves a lower triangular system. Moreover, the computational cost for the evaluation of the integrals of the form $\int_{a}^{x_{0}}\left(x_{n}-s\right)^{-\alpha} K\left(x_{n}, s, y(s)\right) d s$, with $x_{0} \equiv b$ fixed, increases as $h \rightarrow 0$; in fact, we need to use the product formula described in $\S 2$ based on the Radau nodes or the Gauss-Radau rule, according to whether $x_{n}-x_{0}<k\left(x_{0}-a\right)$ or $x_{n}-x_{0} \geq k\left(x_{0}-a\right)$ respectively, where $k$ is a constant which we can find by numerical experiments. (For example, for various values of $\alpha$ and $N=32$ we have found $k \simeq 1 / 20$.) In practice, wecould choose $b$ depending on $h$ and assume $b-a=h / k$ (with an ap- 
propriate $k$ ), taking advantage of the fact that this allows a reduction in the global cost since, for each $h$, we can evaluate $g\left(x_{n}\right)$, for any $n$, using a Gaussian rule with low $N$. It is this last version that we have implemented.

In order to test the performance of our method, we have compared our numerical results with the corresponding ones available in the literature.

Special attention is given to the results produced by the computer program given in [17]; in fact, the fractional linear multistep methods introduced by Lubich [24] appear to represent the most promising approach for the efficient solution of Abel equations, as it has been already remarked in [5, p. 496], [7, p. 590].

In the tables below we denote by $P S$ our method, by $P$ the starting method (8), and by $L$ the method implemented in [17].

In Table 1 we denote by $B E$ results concerning the equation (23) presented in [8] and obtained by a collocation technique in certain piecewise polynomial spaces employing uniform meshes. Our method $P S$ has been applied with $b=0.2, h=$ 0.01 .

TABLE 1. Absolute errors for the approximate solution of the equation (23) at $x=1$ computed by different methods

\begin{tabular}{|r|l|l|c|c|}
\hline$N$ & \multicolumn{1}{|c|}{$P S$} & \multicolumn{1}{|c|}{$P$} & $B E$ & $L$ \\
\hline 4 & $8.7 \mathrm{D}-8$ & $4.3 \mathrm{D}-5$ & & $3.4 \mathrm{D}-8$ \\
5 & $1.0 \mathrm{D}-8$ & $1.8 \mathrm{D}-5$ & $8.4 \mathrm{D}-4$ & \\
8 & $2.1 \mathrm{D}-9$ & $7.3 \mathrm{D}-7$ & & $4.1 \mathrm{D}-8$ \\
10 & $8.7 \mathrm{D}-10$ & $1.2 \mathrm{D}-7$ & & \\
16 & $1.0 \mathrm{D}-10$ & $6.6 \mathrm{D}-9$ & & $2.3 \mathrm{D}-8$ \\
20 & $3.6 \mathrm{D}-11$ & $8.6 \mathrm{D}-10$ & $5.0 \mathrm{D}-5$ & \\
32 & $3.8 \mathrm{D}-12$ & $1.1 \mathrm{D}-10$ & & $4.7 \mathrm{D}-9$ \\
\hline
\end{tabular}

Concerning the equation (24), Table 2 shows results taken from: [21] (denoted by $L Z$ and obtained by a product integration method of order $p=3$ based on generalized Simpson's rule); from [15] (denoted by ET and obtained by a sixth-order method using spline functions of degree 5 , deficiency 4 , i.e., in the continuity class $C$, on uniform meshes); from [12] (denoted by $C K$ and obtained by an interpolatory product integration method of order $p=5$, on uniform meshes, which is an extension of the approach proposed in [19]); from [29] (denoted by $R$ and obtained by collocation type methods).

Notice that the convergence results obtained by the methods $L Z, E T, C K$ are only valid if the solution $y(x)$ is sufficiently smooth.

Our method PS has been implemented with $b=0.02, h=0.002$.

The results denoted by $P S$ in Table 3 have been obtained with $b=0.1, h=0.01$.

In the literature we were able to find only numerical examples concerning kernels of type (i). Table 4 shows results obtained by application of the method $P$ to the equation

$$
\begin{aligned}
& y(x)=0.1\left\{1-e^{-\left(\frac{x+1}{2}\right)}-\frac{1}{\sqrt{2 \pi}} \int_{-1}^{x} \log |x-s| y(s) d s\right\}, \\
& y(x)=0.1\left\{1-e^{-\left(\frac{x+1}{2}\right)}\right\}+O\left[(x+1)^{2} \log ^{2}(x+1)\right] \quad \text { as } x \rightarrow 1 .
\end{aligned}
$$

The asymptotic behavior of the solution of the equation is deduced from [22, Corollary 3.2]. 
TABLE 2. Absolute errors for the approximate solution of the equation (24) at $x=1$ computed by different methods

\begin{tabular}{|r|l|l|c|c|c|c|c|}
\hline$N$ & \multicolumn{1}{|c|}{$P S$} & $P$ & $L Z$ & \multicolumn{1}{c|}{$E T$} & $C K$ & $R$ & $L$ \\
\hline 4 & $1.4 \mathrm{D}-9$ & $2.8 \mathrm{D}-6$ & & $\leq 7.0 \mathrm{D}-8$ & & & $3.0 \mathrm{D}-7$ \\
5 & $1.9 \mathrm{D}-10$ & $3.1 \mathrm{D}-7$ & $5.6 \mathrm{D}-6$ & & & D-8.49 & \\
8 & $9.7 \mathrm{D}-13$ & $6.3 \mathrm{D}-10$ & & $\leq 2.0 \mathrm{D}-9$ & & & $1.3 \mathrm{D}-7$ \\
10 & & $1.2 \mathrm{D}-11$ & $8.0 \mathrm{D}-7$ & & & D-8.6 & \\
16 & & $1.3 \mathrm{D}-16$ & & & & & $1.4 \mathrm{D}-8$ \\
20 & & & $1.0 \mathrm{D}-7$ & & & D-9.33 & \\
32 & & & & & & & $1.9 \mathrm{D}-9$ \\
64 & & & & & & & \\
100 & & & & & $2.8 \mathrm{D}-14$ & & \\
128 & & & & & $5.6 \mathrm{D}-16$ & & \\
200 & & & & & & & \\
256 & & & & & & & $5.7 \mathrm{D}-11$ \\
\hline
\end{tabular}

TABLE 3. Absolute errors for the approximate solution of the equation (25) at $x=8$. The exact solution at $x=8$ is $y(8)=$ $0.3236412904[24]$

\begin{tabular}{|r|c|c|c|}
\hline$N$ & $P S$ & $P$ & $L$ \\
\hline 4 & $6.4 \mathrm{D}-9$ & $3.9 \mathrm{D}-2$ & $6.7 \mathrm{D}-2$ \\
8 & & $3.0 \mathrm{D}-3$ & $2.2 \mathrm{D}-2$ \\
16 & & $2.8 \mathrm{D}-4$ & $2.7 \mathrm{D}-3$ \\
32 & & $5.2 \mathrm{D}-6$ & $8.6 \mathrm{D}-6$ \\
64 & & $6.9 \mathrm{D}-9$ & $1.0 \mathrm{D}-5$ \\
128 & & & $1.9 \mathrm{D}-6$ \\
256 & & & $7.3 \mathrm{D}-8$ \\
\hline
\end{tabular}

TABLE 4. Absolute errors for the approximate solution of the equation (27) at $x=1$ computed by the method $P$

\begin{tabular}{|r|l|}
\hline$N$ & $P$ \\
\hline 4 & $3.3 \mathrm{D}-8$ \\
8 & $3.2 \mathrm{D}-10$ \\
16 & $5.2 \mathrm{D}-13$ \\
\hline
\end{tabular}

An inspection of the above numerical experiments shows that the numerical results we obtained by our method in the linear cases confirm the theoretical rate of convergence. Moreover, our method can provide reasonable results for nonlinear as well as linear problems. In fact, for both kinds of test equations we considered, the convergence properties of the method $P S$ appear similar or superior to the ones of the other methods considered.

Concerning the computational efficiency of our method PS, we compare its computational cost with the one of the numerical procedure $L$ presented in [17], which, 
among the methods we have found in the literature, gives the best results, in particular for the equations with nonsmooth solutions. Whereas our starting method $P$ requires the solution of the $(N+1) \times(N+1)$ system (8) in addition to $O\left(N^{2}\right)$ arithmetical operations to compute the quadrature weights, the algorithm proposed by Lubich requires $O(N \log N)$ arithmetical operations to compute the quadrature weights and $O\left[N(\log N)^{2}\right]$ arithmetical operations to obtain approximations to the solution at the grid points by FFT techniques; see [10, (§6.1)], [17, 18]. Nevertheless, in the case of the equation (23) (see Table 1) the methods $P S$, with $[a, b]=[0 ., 0.2], h=0.01$, and $L$ involve $N=8$ nodes (and a timing of 0.02 seconds) and $N=32$ nodes (and a timing of 0.03 seconds), respectively, to compute the approximate solution at $x=1$ with an absolute error of order of magnitude $10^{-9}$ * $^{*}$

The behavior of the errors in Table 2 is due to the fact that the solution of the equation (24) is smooth in the closed interval of integration. In particular, we can see that if the equation (24) is solved over the range $0 \leq x \leq 0.02$ by the starting method $P$, the method $P S$, with $h=0.002$, requires $N=8$ and a timing of 0.18 seconds to compute the approximate solution at $x=1$ with an absolute error of order of magnitude $10^{-12}$. The same accuracy for the approximate solution at $x=1$ is obtained by the method $L$ with $N=256$ and a timing of 0.18 seconds.* $^{*}$ On the other hand, if we apply our starting method $P$ on the whole interval [0., 1.] the full accuracy at $x=1$ requires $N=16$ and a timing of 0.01 seconds.

We conclude that our starting method together with an appropriate step-bystep method for regular solutions appears to be a useful tool for the numerical computation of the solutions of Volterra integral equations of the second kind with weakly singular kernels.

The numerical results presented have been obtained on a VAX 9000 computer working with about 16-digit double-precision arithmetic.

\section{ACKNOWLEDGMENT}

I am grateful to Professor S. Prössdorf for his helpful suggestions which, in particular, led to simple proofs of the compactness of the operator $A$ and of the Lemma 3.

\section{REFERENCES}

1. J. Abdalkhani, A numerical approach to the solution of Abel integral equations of the second kind with nonsmooth solution, J. Comput. Appl. Math. 29 (1990), 249-255. MR 91h:65216

2. P. M. Anselone, Collectively compact operator approximation theory and applications to integral equations, Prentice-Hall, Englewood Cliffs, NJ, 1971. MR 56:1753

3. K. E. Atkinson, An existence theorem for Abel integral equations, SIAM J. Math. Anal. 5 (1974), 729-736. MR 51:3822

4. C. T. H. Baker, The numerical treatment of integral equations, Clarendon Press, Oxford, 1977. MR 57:7079

5. C. T. H. Baker, The state of the art in the numerical treatment of integral equations, The State of the Art in Numerical Analysis, Clarendon Press, Oxford, 1987, pp. 473-509. MR 88m:65200

6. H. Brunner, The numerical solution of integral equations with weakly singular kernels, Numerical Analysis (D. F. Griffiths, ed.), Lecture Notes in Math., vol. 1066, Springer-Verlag, Berlin, 1984, pp. 50-71. MR 85j:65043

\footnotetext{
*The results of the method $L$ have been obtained by the Fortran program published in [17].
} 
7. H. Brunner, Collocation methods for one-dimensional Fredholm and Volterra integral equations, The State of the Art in Numerical Analysis, Clarendon Press, Oxford, 1987, pp. 563-600. MR 89m:65112

8. H. Brunner and M. D. Evans, Piecewise polynomial collocation for Volterra-type integral equations of the second kind, J. Inst. Math. Appl. 20 (1977), 415-423. MR 57:14540

9. H. Brunner and H. J. J. te Riele, Volterra-type integral equations of the second kind with nonsmooth solutions, J. Integral Equations 6 (1984), 187-203. MR 85h:65268

10. H. Brunner and P. J. van der Houwen, The numerical solution of Volterra equations, NorthHolland, Amsterdam, 1986. MR 88g:65136

11. B. Cahlon, Numerical solution of non-linear Volterra integral equations, J. Comput.Appl. Math. 7 (1981), 121-128. MR 83b:65147

12. R. F. Cameron and S. McKee, Product integration methods for second kind Abel integral equations, J. Comput. Appl. Math. 11 (1984), 1-10. MR 85k:65104

13. G. Criscuolo, G. Mastroianni, and G. Monegato, Convergence properties of a class of product formulas for weakly singular integral equations, Math. Comp. 55 (1990), 213-230. MR 90m: 65230

14. J. Dixon, On the order of the error in discretization methods for weakly singular second kind Volterra integral equations with non-smooth solutions, BIT 25 (1985), 624-634. MR 86m:65160

15. M. E. A. El Tom, Spline function approximations to the solution of singular Volterra integral equations of the second kind, J. Inst. Math. Appl. 14 (1974), 303-309.

16. R. Gorenflo and S. Vessella, Abel integral equations, a historico-bibliographical survey, IAGA, Firenze, 1984.

17. E. Hairer, Ch. Lubich, and M. Schlichte, Fast numerical solution of weakly singular Volterra integral equations, J. Comput. Appl. Math. 23 (1988), 87-98. MR 89g:65166

18. P. Henrici, Applied and computational complex analysis, vol. 3, Wiley, New York, 1986. MR 87h:30002

19. F. R. de Hoog and R. Weiss, High order methods for a class of Volterra integral equations with weakly singular kernels, SIAM J. Numer. Anal. 11 (1974), 1166-1180. MR 51:4699

20. B. Kress, Linear integral equations, Springer-Verlag, Berlin, 1989. MR 90j:45001

21. P. Linz, Numerical methods for Volterra integral equations with singular kernels, SIAM J. Numer. Anal. 6 (1969), 365-374. MR 41:4850

22. J. E. Logan, The approximate solution of Volterra integral equations of the second kind, $\mathrm{PhD}$ thesis, University of Iowa, Iowa City, 1976.

23. Ch. Lubich, Runge-Kutta theory for Volterra and Abel integral equations of the second kind, Math. Comp. 41(1983), 87-102. MR 85a:65178

24. Ch. Lubich, Fractional linear multistep methods for Abel-Volterra integral equations of the second kind, Math. Comp. 45 (1985), 463-469. MR 86j:65181

25. G. Mikhlin and S. Prössdorf, Singular integral operators, Springer-Verlag, Berlin, 1986. MR 88e: 47097

26. G. Monegato, Orthogonal polynomials and product integration for one-dimensional Fredholm integral equations with "nasty" kernels, Problems and Methods in Mathematical Physics (F. Kuhnert, B. Silbermann, eds.), 9.TMP, Teubner-Texte zur Mathematik, Band 111, Leipzig, 1989, pp. 185-192. CMP 91:06

27. P. Nevai, Mean convergence of Lagrange interpolation. III, Trans. Amer. Math. Soc. 282 (1984), 669-698. MR 85c:41009

28. R. Piessens, Modified Clenshaw-Curtis integration and applications to numerical computation of integral transforms, Numerical Integration, Nato ASI Series, Series C, vol. 203, Reidel, Dordrecht, 1987. MR 88j:65050

29. H. J. J. te Riele, Collocation methods for weakly singular second kind Volterra integral equations with non-smooth solution, IMA J. Numer. Anal. 2 (1982), 437-449. MR 84g:65167

30. I. H. Sloan, Analysis of general quadrature methods for integral equations of the second kind, Numer. Math. 38 (1981), 263-278. MR 82m:65128

31. D. Westreich and B. Cahlon, Numerical solution of Volterra integral equations with continuous or discontinuous terms, J. Inst. Math. Appl. 26 (1980), 175-186. MR 82a:65101

Dipartimento di Matematica, Politecnico di Torino, Corso Duca degli Abruzzi, 24, I-10129 TORINO, ITALY

E-mail address: orsi@polito.it 